\title{
Relationship between Sensitivity and Transmittance of Novolac Resist Exposed to Light Emitting Diodes
}

\author{
Toshiyuki Horiuchi ${ }^{1,2}$, Takuya Fujii ${ }^{1}$, Ken Nakamura ${ }^{1}$, Hiroshi Hirano ${ }^{1}$, \\ Koichi Ishikawa ${ }^{2}$ and Jun-ya Iwasaki ${ }^{2}$ \\ ${ }^{1}$ Tokyo Denki University \\ ${ }^{2}$ Graduate school of Engineering, Tokyo Denki University \\ 2-2, Kanda-Nishiki-cho, Chiyoda-ku, Tokyo, 101-8457, Japan \\ *Present address: Cottrell Engineering Co., Ltd.
}

\begin{abstract}
Sensitivity of the novolac positive resist THMR iP-3300 to violet and blue light emitting diodes with central wavelengths of $404-470 \mathrm{~nm}$ was investigated to utilize for developing a new numbering exposure system using a matrix array of such light emitting diodes. In addition, the spectral transmittance of the resist was measured, and the relationship between transmittance and sensitivity was discussed. It was clarified that the sensitivity greatly differed between exposures to violet and blue light rays as much as more than 30 times, and the resist was sensitized only by $3 \mathrm{~s}$ exposure to the violet light at minimum. Therefore, reasonable exposure time performance will be obtained if the relevant reduction projection lens is applied.
\end{abstract}

Keywords: novolac resist, spectral transmittance, sensitivity, light emitting diode

\section{Introduction}

Guarantee of industrial product performance is one of the most important measures for a maker to get reliance of customers. For this reason, every maker company manages the quality by assigning an individual serial number and/or a code mark for each product component. If a 2-dimensional code mark is printed on each component, information such as fabrication conditions, lot numbers, workers, test results of initial performance, and others are compactly recorded.

Two types of numbering exposure tool or code mark exposure tool are commercialized by Toray Engineering and others. One is a projectionexposure type, and the other is a laser-scan type. In the former, a small liquid crystal display (LCD) is used as a reticle, and numbers and code marks assigned on the LCD are projected onto the product components such as substrates, panels and plates coated with resist films. In the latter, numbers and code marks are directly delineated.

If an exposure system utilizing a matrix array of light emitting diodes (LEDs) in place of the LCD reticle and an illumination system, expensive exposure light source such as a mercury lamp and a laser is not necessary. However, the LED should be appropriately selected to suppress the exposure time reasonably short and print fine and clear code marks. Generally speaking, projected pattern images through a projection lens are formed more clearly using exposure light rays with a shorter wavelength. However, the patterning performances also depends on the resist characteristics, and sharp pattern edges are not always obtained even if the light images of the patterns are clearly formed with a high contrast. If transmittance of resist is too low, exposure light rays do not penetrate the resist film. Accordingly, the bottom parts of the resist film are not sufficiently sensitized, and the patterns are not 
completely resolved. On the other hand, when a resist has too high transmittance, most of the exposure light rays pass through the resist films without being absorbed. For this reason, the resist films are hardly sensitized, and long exposure time is needed to print patterns.

From these points of view, it is very important to select the exposure wavelength matching the resist used in the fabrication processes of the products. For this reason, relationship between the spectral transmittance and sensitivity is investigated here using LED candidates on the market and a versatile novolac-type i-line resist.

\section{Spectral Transmittance}

At first, spectral transmittance of a resist was investigated. As a resist, positive novolac-type THMR iP-3300 (Tokyo Ohka Kogyo) was used. Because this resist had bleaching characteristics, change of transmittance according to the exposure dose was also investigated. Quartz plates with a size of $50 \mathrm{~mm}$ square and a thickness of $1 \mathrm{~mm}$ were coated with approximately $1-\mu \mathrm{m}$ thick resist films, and they were exposed using an ultra violet lamp (Sumita Optical Glass, LS-140), changing the exposure time variously to control the exposure dose. The light spectrum of the lamp was measured using a spectral intensity meter (Ocean Optics, HR400CG- UV-NIR), as shown in Fig. 1.

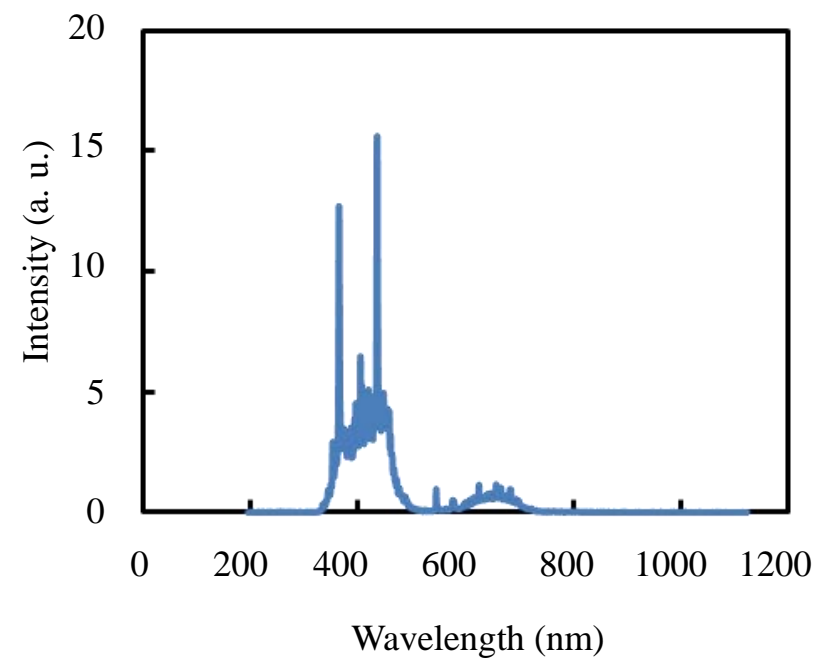

Fig. 1. Light spectrum of ultra violet lamp used for investigating bleaching characteristics.
The spectral transmittance was measured before and after the exposure using a spectrophotometer (JASCO, V-630) between the wavelengths of 200-800 $\mathrm{nm}$. The fully transparent light intensity level (transmittance of 1.00) was calibrated by using the quartz plate without the resist film. Measured transmittance curves are shown in Fig. 2. It is known from the Figure that the bleaching occurs for a wide wavelength range of 300-440 nm. Although the transmittances much differ before the exposure, they become almost same level after the sufficient exposure. These curves and the bleaching trends were similar to those reported for the other novolac resists. [1][2] It is expected from the Figure that high sensitivity is obtained for wavelengths at which the transmittances largely change before and after the exposure, because the bleaching occurs when photoactive diazonaphthoquinone (DNQ) absorbs the exposure light.

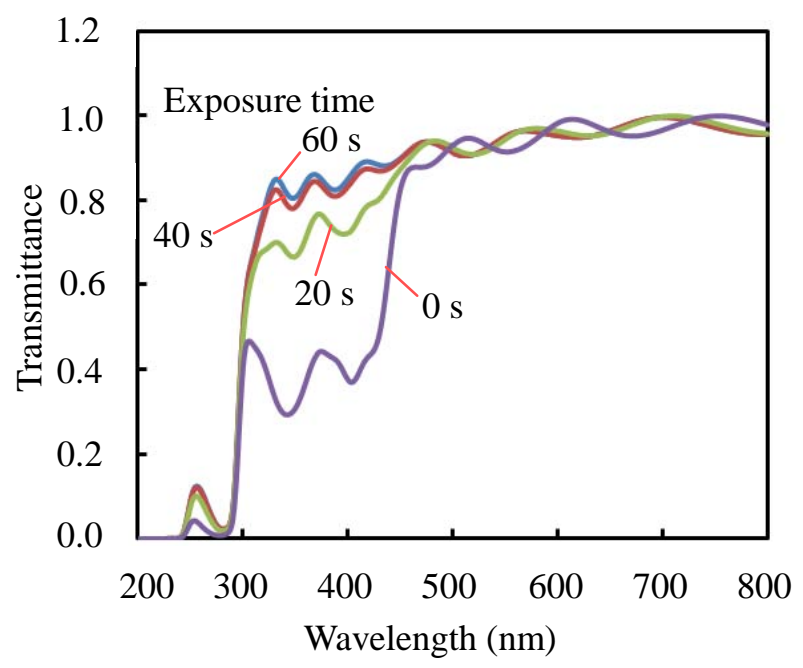

Fig. 2. Spectral transmittance of THMR iP-3300.

\section{Spectral Sensitivity}

Next, spectral sensitivity was investigated using LEDs. The speed of the photochemical reaction in the resist relates to the exposure light power absorbed in it, and the absorption relates to the bleaching characteristics. Accordingly, it is considered that the light rays with wavelengths at which remarkable bleaching effects were observed are strongly absorbed in the resist. For this reason, sensitivity difference was investigated using LEDs 
with wavelengths of 404-470 nm. Roughly speaking, the colors of the light with these wavelengths are violet and blue.

At first, the spectra of LEDs were measured using a spectral intensity meter (Ocean Optics, HR400CG- UV-NIR) and simple experimental setups, as shown in Fig. 3.

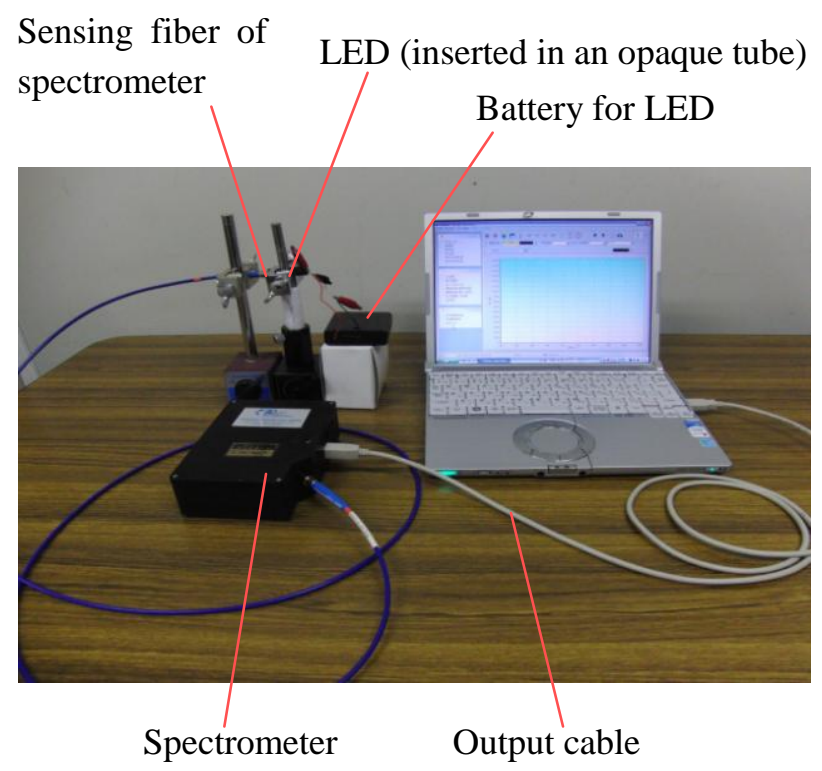

Fig. 3. Setups for measuring LED spectrum.

The fiber entrance of the spectral intensity meter and the LED were covered by a black rubber tube so as noise light rays are not sensed. All of the LEDs used in the experiments had a bullet shape with a diameter of $5 \mathrm{~mm}$. Measured spectra are shown in Fig. 4.

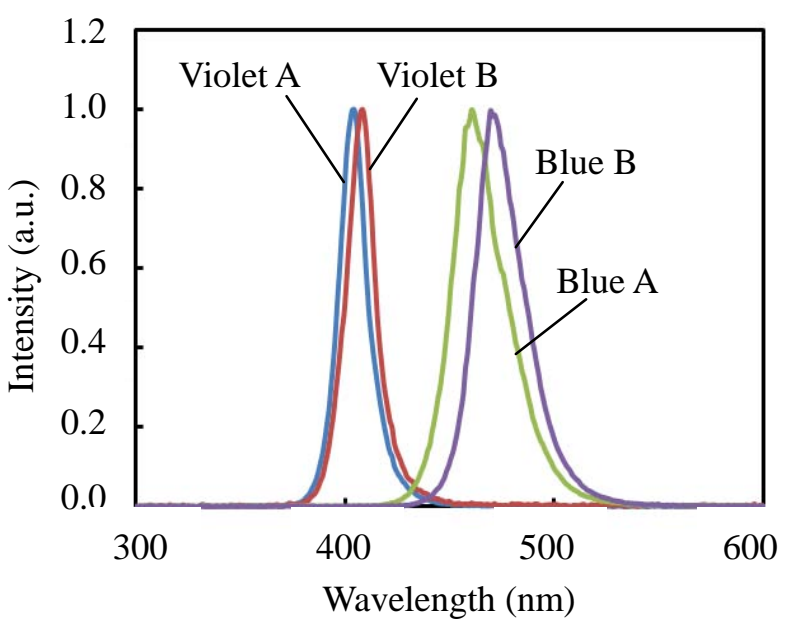

Fig. 4. Spectral emission curves of LEDs.
The central wavelength and the full width half maximum (FWHM) for each LED were listed in Table 1.

Although the spectra had slightly wide distributions, it was judged that the distributions were narrow enough to compare the sensitivity between violet and blue light rays with wavelengths shown in Table 1.

Table 1. Light emitting diode used for experiments.

\begin{tabular}{ccc}
\hline LED & $\begin{array}{c}\text { Central Wavelength } \\
(\mathrm{nm})\end{array}$ & $\begin{array}{c}\text { FWHM } \\
(\mathrm{nm})\end{array}$ \\
\hline Violet A & 404 & 15 \\
Violet B & 408 & 15 \\
Blue A & 460 & 29 \\
Blue B & 470 & 27 \\
\hline
\end{tabular}

Using these 4 types of LEDs, the sensitivity of the resist THMR iP-3300 was investigated. The resist was coated on 4-inch silicon wafers in approximately $1 \mu \mathrm{m}$ thick. Figure 5 shows the schematic structure of the simple exposure tool used for the experiments. The LEDs were attached one by one to the tool. The LED was fixed in the center of the black resin plate, and the light rays were only ejected through a small outlet hole with a diameter $d$ of $1.1 \mathrm{~mm}$. This small hole was almost contacted to the resist surface.

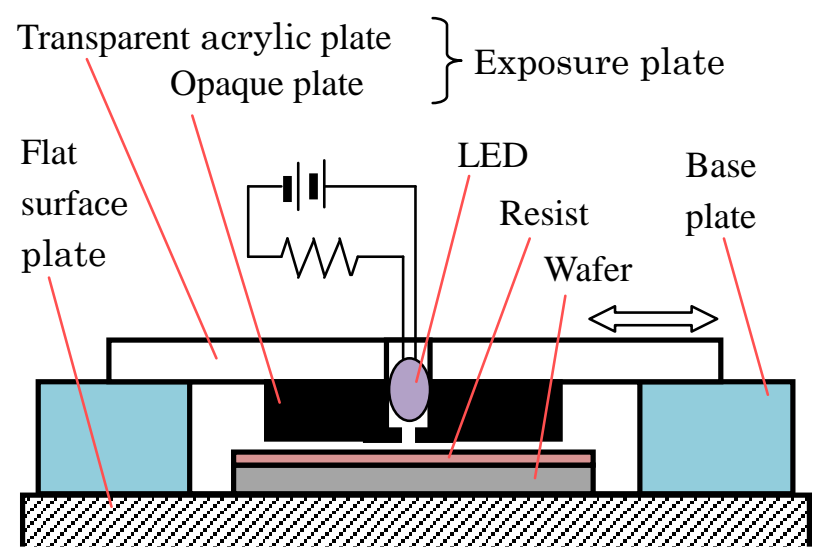

Fig. 5. Schematic structure of exposure tool.

Before the exposure, the light power $P$ emitted from the small hole was measured using a power meter (Sanwa Electric Instrument, LP1). After compensating the read-out power value by an 
appropriate coefficient considering the wavelength, the power $P$ was converted to the light intensity $I$ per unit area using eq. (1).

$$
I=\frac{P}{\frac{\pi}{4} d^{2}} .
$$

The tool was handmade, and Figure 6 shows a plane-view photograph. The exposure plate, in the center of which the LED was attached, was made of a transparent acrylic plate and an opaque plate surrounding the LED, and was placed on the base frame with scales in $\mathrm{X}$ and $\mathrm{Y}$ directions. The exposure position was decided by referring to the scales and manually moving the exposure plate step by step. The LEDs were lit using dry batteries with a voltage of $6 \mathrm{~V}$.

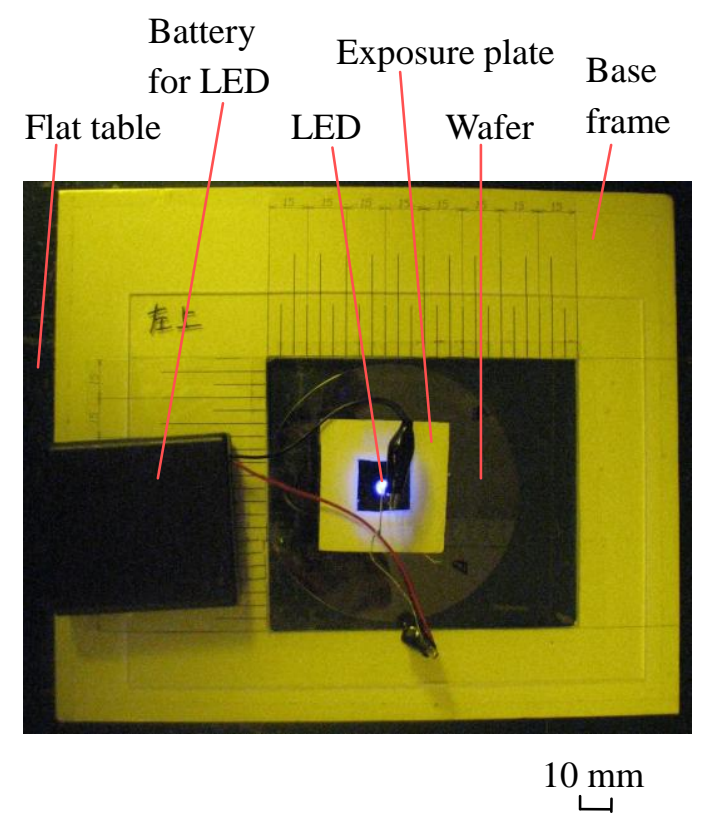

Fig. 6. Plane view of the LED-exposure tool.

The resist was exposed step by step changing the exposure time, and the diameter $D$ was measured for each hole-pattern. The changes of hole-pattern sizes are shown in Fig. 7.

Threshold exposure time $t_{\mathrm{s}}$ to sensitize the resist was judged from this graph. Using the critical sensitization time $t_{s}$ for each LED, the resist sensitivity $S$ was calculated by

$$
S=I \cdot t_{s} .
$$

Calculated $I$ and $S$ based on the experimental results are shown in Table 2, and the relationship between the sensitivity $S$ and central wavelength of the LED is shown in Fig. 7.

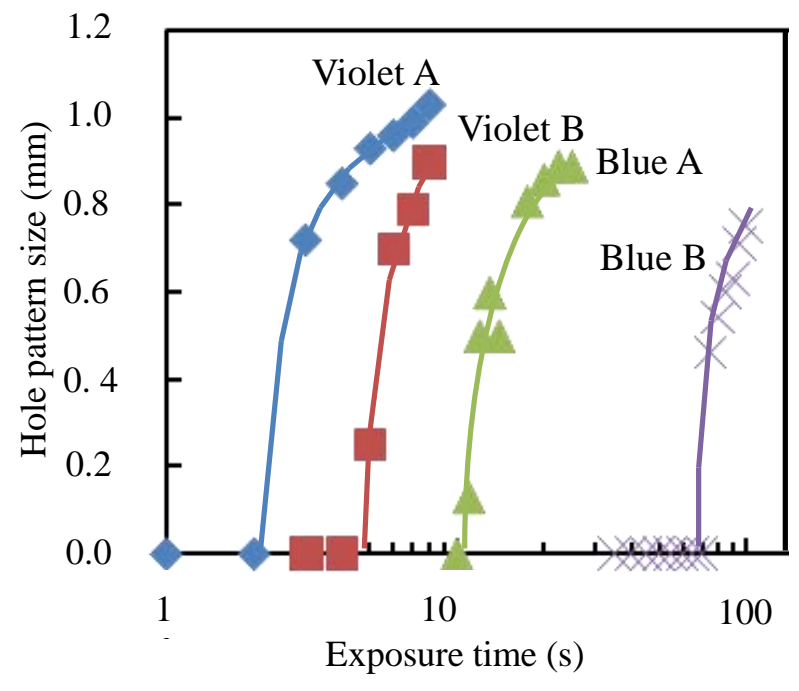

Fig. 7. Relationship between hole-pattern size and the exposure time.

Table 2. Measured intensity of LEDs and surmised sensitivity of the resist.

\begin{tabular}{lccc}
\hline LED & $\begin{array}{c}\text { Intensity } I \\
\left(\mu \mathrm{W} / \mathrm{mm}^{2}\right)\end{array}$ & $\begin{array}{c}\text { Time } t_{\mathrm{s}} \\
(\mathrm{s})\end{array}$ & $\begin{array}{c}\text { Sensitivity } S \\
\left(\mathrm{~mJ} / \mathrm{cm}^{2}\right)\end{array}$ \\
\hline Violet 1 & 216 & 2.5 & 54 \\
Violet 2 & 96 & 6 & 58 \\
Blue 1 & 420 & 13 & 546 \\
Blue 2 & 246 & 75 & 1850 \\
\hline
\end{tabular}

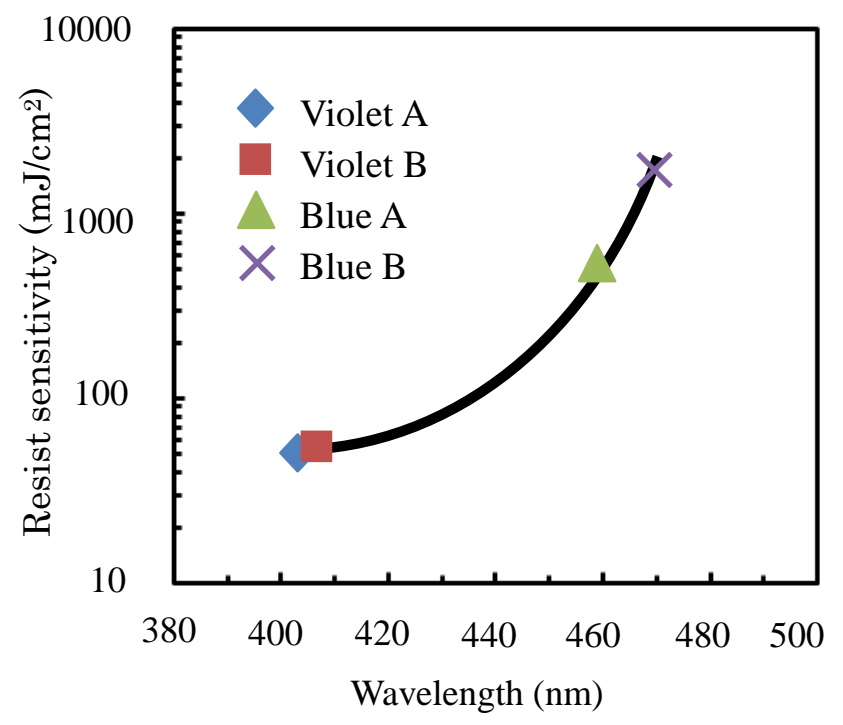

Fig. 8. Resist sensitivity dependence on exposure wavelength. 
It was clarified that sensitivity strongly relates to absorption, as having been reported, [3] and sensitivity for violet light with wavelengths of $404 \mathrm{~nm}$ was more than 30 times higher than that for blue light with wavelengths of $470 \mathrm{~nm}$.

\section{Relationship between Transmittance and Sensitivity}

Relationship between the transmittance and the sensitivity was discussed. From Fig. 1, the initial transmittance before the exposure for 404-nm light and 470-nm light are 0.37 and 0.87 , respectively. The transmittance values increased to 0.88 and 0.94 after giving sufficient exposure dose of $60 \mathrm{~s}$.

It was supposed at the beginning that the absorbed energy was roughly proportional to the transmittance change. However, the transmittance changes between before and after the exposure were 0.50 and 0.06 , respectively, and the ratio of $0.50 / 0.06=8.3$ is much smaller than the sensitivity change of more than 30 times. The reason why the sensitivity varies much larger than the transmittance was considered to be the following two.

When a photon is absorbed in a molecule of the resist, transmittance increases depending on the bleaching effect. However, the transmittance simultaneously varies for a wide wavelength range without depending what energy has a photon which contributes the bleaching phenomena. Because the transmittance change is caused by the decomposition of DNQ, the transmittance varies for all the wavelengths if the DNQ is only decomposed by the light with some particular wavelengths. The spectral transmittance change shown in Fig. 2 was mainly caused by the light with wavelengths of $350-460 \mathrm{~nm}$, especially by the bright lines of 365,405 and $436 \mathrm{~nm}$ judging from the spectral intensity distribution of the UV lamp, as shown in Fig. 1. The spectral transmittance change was not caused by each single wavelength light corresponding to each spectrum. This is one reason.

The other reason is the weight percentage of the photoactive compound DNQ. Referring to the literature, [4] DNQ is contained roughly $20 \mathrm{wt} \%$ in the novolac resist, and the novolac base resin is not completely transparent for the exposure light. This is known from the fact that the transmittance does not reach to 1.00 even after sufficiently giving the dose. For this reason, the transmittance of the resist is only partially influenced by the change of the DNQ transmittance.

As explained above, although the sensitivity differences for different wavelengths were not quantitatively predicted, it was clarified that the trends of spectral sensitivity certainly corresponded to the spectral transmittance of resist. Because the spectral transmittance of resist is easily and simply measured, it would be a good criterion to judge the sensitivity.

Recently, various new LEDs emitting light with wavelengths shorter than $400 \mathrm{~nm}$ are eagerly developed.[5]-[8] If these LEDs are commercially available and the prices are reduced down, the sensitivity for these short-wavelength LEDs can be investigated, and the relationship between transmittance and sensitivity may be much more clarified.

On the other hand, because the resist was sensitized within $3 \mathrm{~s}$ using an LED with a wavelength of $404 \mathrm{~nm}$, It was supposed that the resist could be sensitized within $100-200 \mathrm{~ms}$ if a highly efficient reduction projection exposure system with a reduction ratio of $1 / 4-1 / 5$ was applied.

\section{Conclusion}

Spectral sensitivity of the novolac resist was evaluated using LEDs with wavelengths of 404-470 $\mathrm{nm}$. The sensitivity difference between these wavelengths was as large as more than 30 times. It was also clarified that the sensitivity differences were predicted to some extent by measuring the spectral transmittance change between before and after the bleaching. Depending on the bleaching effect, the transmittance varies in proportion to the absorption of exposure light into the photoactive compound DNQ.

However, because the novolac base resin is not completely transparent, the increase of the transmittance caused by the reaction of PAC is apparently suppressed. In addition, measured change of the spectral transmittance does not 
caused by each single wavelength light for each spectrum. For these reasons, the change of the transmittance does not directly concern to the sensitivity.

It has been reported that the light absorption by the resist strongly influences cross-sectional profiles and edge roughness of patterns besides the sensitivity. [9]-[11] Accordingly, it is anticipated that the wavelength also influences them. For this reason, dependence of the cross-sectional profiles and edge roughness of patterns on the wavelength and bleaching conspicuousness should be investigated in detail hereafter.

\section{Acknowledgement}

This work was partially supported by Research Institute for Science and Technology of Tokyo Denki University Grant Number Zc08-01 in 2008 and Q09M-05 in 2009.

\section{References}

1. M. Endo, M. Sasago, A. Ueno, and N. Nomura, J. Vac. Sci. Technol. B 7 (1989) 565.

2. H. Namatsu, J. Vac. Sci. Technol. B 6 (1988) 1698.

3. S. Rice and K. Jain, IEEE Trans. Electron Devices, 31, 1 (1984) 1.
4. H. Ito, Resist zairyo (Resist materials) (Kyoritsu Shuppan, Tokyo, 2005) p. 14 [in Japanese].

5. D. Morita, M. Sano, M. Yamamoto, T. Murayama, S. Nagahama, and T. Mukai, Jpn. J. Appl. Phys. 41 (2002) L1434.

6. T. Wang, Y. H. Liu, Y. B. Lee, J. P. Ao, J. Bai, and S. Sakai, Appl. Phys. Lett., 81 (2002) 2508.

7. K. Mayes, A. Yasan, R. McClintock, D. Shiell, S. R. Darvish, P. Kung, and M. Razeghi, Appl. Phys. Lett., 84 (2004) 1046.

8. A. J. Fischer, A. A. Allerman, M. H. Crawford, K. H. A. Bogart, S. R. Lee, R. J. Kaplar, W. W. Chow, S. R. Kurtz, K. W. Fullmer, and J. J. Figiel, Appl. Phys. Lett., 84 (2004) 3394.

9. T. Horiuchi and T. Kashiwagi, Microelectronic Engineering 87 (2010) 977.

10. Y. Tomo, T. Kasuga, M. Saito, A. Someya, and T. Tsumori, Vac. Sci. Technol. B 10 (1992) 2576.

11. A. Y. B. M. Bakri, M. J. Manaf, K. A. Wahab, M. I. B. Ahmad, Int. Electronic Manufacturing Tech., Putrajaya, Malaysia (2006) 416. 